\title{
ON THE EVERTED STATE OF SPHERICAL AND CYLINDRICAL SHELLS
}

\author{
BY
}

\author{
ANDREW J. SZERI
}

California Institute of Technology, Pasadena, California

\begin{abstract}
The existence, uniqueness, and qualitative nature of the everted state of spherical and cylindrical shells is examined for elastic materials with stress functions satisfying relatively simple restrictions. Qualitative analysis of the governing nonlinear ordinary differential equation is facilitated by recasting it in stress-stretch coordinates. One finds a continuum of solutions which meet the boundary conditions. Each corresponds uniquely to a shell of a particular thickness. We present numerical solutions which demonstrate the claims of the analysis.
\end{abstract}

1. Introduction. The problem of eversion of shells of elastic materials is of considerable interest because it is a paradigm of multiplicity of solutions in elasticity. That one can turn "inside out" a rubber hose or hollow ball is a matter of common experience, but the mathematical description of a unique everted state has eluded the efforts of researchers, except in the more simple case of incompressible elastic materials where the complete solution is known.

The question of eversion was identified and formulated by Green and Adkins [2] and by Truesdell and Noll [6] who also present the solution to the incompressible problem. Antman [1] treats the problem of the eversion of thick spherical shells from a functional analytic viewpoint. The existence, but not the uniqueness, of an everted state is proved in [1], subject to hypotheses on the stress response function. Following Hill [3], we find sufficient conditions for uniqueness of the everted state based on the form of the stress response function.

In this paper, we attack the problem of eversion of spherical and cylindrical shells of elastic materials from a qualitative theory of differential equations point of view. These methods have been used by other researchers (see Sivaloganathan [5], among others). When viewed in a mixture of stress and principal stretch variables, one finds that the governing differential equation (of static equilibrium) is amenable to simple but powerful qualitative analysis. The required constitutive restrictions on the stress functions are strong ellipticity, natural growth conditions, and positivity of a discriminant of the stress functions, which we define and interpret below. In the case of hyperelastic materials, the latter condition is equivalent to requiring the

Received June 28, 1988.

(C) 1990 Brown University 
positive definiteness of the Hessian of the work function with respect to purely radial (and everted) deformations.

In Sec. 2 we present the analysis which establishes existence and uniqueness for the everted spherica! shell. Perhaps more importantly, the analysis provides a qualitative description of the stress distribution through the thickness of the shell. At the end of the section, we give numerical results of a particular example of an Ogden material to demonstrate that the everted shell behaves as the analysis predicts. The analysis of cylindrical shells is quite similar; we present an abbreviated solution of that problem in Sec. 3. Finally, we give our conclusions in Sec. 4.

2. Eversion of spherical shells. In this section, we shall prove the following

THEOREM 1. Given a spherical shell of an elastic material, there is a unique everted state for the shell provided that the stress functions of the material satisfy the strong ellipticity condition, the appropriate growth conditions, and have a strictly positive discriminant.

First, we give the formulation of the governing equations. Then we proceed to perform qualitative analysis to establish the claim of Theorem 1. We give various comments, where appropriate, for specialization of the analysis to the case of hyperelastic materials.

Formulation. Following Antman [1], we formulate the governing equation for a spherical shell undergoing eversion. Let $\mathbf{B}$ be the body in the reference configuration,

$$
\mathbf{B}=\left\{(r, \theta, \phi): r_{i} \leq r \leq r_{o},-\pi \leq \theta \leq \pi, 0 \leq \phi \leq 2 \pi\right\},
$$

where $(r, \theta, \phi)$ are spherical polar coordinates assigned to positions in Euclidean threespace. We have the orthonormal basis vectors

$$
\begin{aligned}
& \mathbf{a}_{1}(\theta, \phi)=\mathbf{a}^{1}(\theta, \phi)=\sin \theta \cos \phi \mathbf{i}+\sin \theta \sin \phi \mathbf{j}+\cos \theta \mathbf{k} \\
& \mathbf{a}_{2}(\theta, \phi)=\mathbf{a}^{2}(\theta, \phi)=\cos \theta \cos \phi \mathbf{i}+\cos \theta \sin \phi \mathbf{j}-\sin \theta \mathbf{k} \\
& \mathbf{a}_{3}(\phi)=\mathbf{a}^{3}(\phi)=-\sin \phi \mathbf{i}+\cos \phi \mathbf{j}
\end{aligned}
$$

for the reference configuration, where $\{\mathbf{i}, \mathbf{j}, \mathbf{k}\}$ is an orthonormal basis for Euclidean three-space. The deformed configuration is described by the orthonormal basis

$$
\begin{aligned}
& \mathbf{b}_{1}(\theta, \phi)=\mathbf{b}^{1}(\theta, \phi)=\sin \theta \cos \phi \mathbf{i}+\sin \theta \sin \phi \mathbf{j}-\cos \theta \mathbf{k} \\
& \mathbf{b}_{2}(\theta, \phi)=\mathbf{b}^{2}(\theta, \phi)=\cos \theta \cos \phi \mathbf{i}+\cos \theta \sin \phi \mathbf{j}+\sin \theta \mathbf{k} \\
& \mathbf{b}_{3}(\phi)=\mathbf{b}^{3}(\phi)=\sin \phi \mathbf{i}-\cos \phi \mathbf{j} .
\end{aligned}
$$

We consider deformations of the form

$$
\mathbf{p}(r, \theta, \phi)=f(r) \mathbf{b}_{1}(\theta, \phi)
$$

where $\mathbf{p}$ is the position of the particle $(r, \theta, \phi)$ in the deformed configuration. Thus the deformation gradient tensor $\mathbf{F}$ is

$$
\mathbf{F}=\frac{d f}{d r} \mathbf{b}_{1} \mathbf{a}^{1}+\frac{f}{r} \mathbf{b}_{2} \mathbf{a}^{2}-\frac{f}{r} \mathbf{b}_{3} \mathbf{a}^{3} .
$$

Anticipating a phase space description, we define the strains $u \equiv d f / d r$ in the radial direction and $v \equiv f(r) / r$ in the $\theta$-direction. The components of the first PiolaKirchhoff stress $\mathbf{S}$ are defined to be $P(u, v)$ in the radial direction, and $Q(u, v)$ in the 
$\theta$-direction. Moreover, $P(1,1)=0$ and $Q(1,1)=0$, i.e., the reference configuration is assumed to be stress-free. The functions $P$ and $Q$ are assumed to be $C^{1}$ in what follows.

In the special case of hyperelasticity, there is a scalar valued work function $\sigma(\mathbf{F})$ related to the stress by

$$
\mathbf{S}=\frac{\partial \sigma}{\partial \mathbf{F}} .
$$

One may define a new work function $\Phi(u, v)=\sigma(\mathbf{F})$ with the properties

$$
\begin{aligned}
& P(u, v)=\frac{\partial \Phi}{\partial u}, \\
& Q(u, v)=\frac{\partial \Phi}{\partial v} .
\end{aligned}
$$

For the shell to be in static equilibrium, the divergence of the stress must vanish. This provides the governing equation for the problem,

$$
\frac{d P}{d r}+\frac{2}{r}(P-Q)=0
$$

as the radial component of the equation of static equilibrium. Furthermore, we require that the spherical faces be traction-free; that is, we require

$$
P(u, v)=0 \quad \text { on } r=r_{i} \text { and } r=r_{o} .
$$

To complete the formulation of the problem, we need an equation relating $u, v$, and $r$ which we obtain by differentiation of $v$

$$
\frac{d v}{d r}=\frac{u-v}{r} .
$$

Finally, we note that $u<0$ and $v>0$ for the mapping $\mathbf{p}$ to preserve the orientation of the reference configuration.

Phase space description. We recast Eqs. (2.6) and (2.7) as a single equation in the $(u, v)$ plane, which we refer to hereafter as the phase plane. Now we apply the chain rule to Eq. (2.6a) and obtain

$$
\frac{\partial P}{\partial u} \frac{d u}{d r}+\frac{\partial P}{\partial v} \frac{d v}{d r}+\frac{2}{r}(P-Q)=0 .
$$

Next, we rearrange this last equation and make use of Eq. (2.7), which yields

$$
\frac{d u}{d r}=\frac{2(Q-P)}{(\partial P / \partial u) r}=\frac{\partial P / \partial v}{\partial P / \partial u}\left(\frac{u-v}{r}\right) .
$$

Note that by hypothesis the elastic material satisfies the strong ellipticity condition, which implies

$$
\frac{\partial P}{\partial u}>0, \text { and } \frac{\partial Q}{\partial v}>0 .
$$

In the case of hyperelasticity, this is equivalent to

$$
\frac{\partial^{2} \Phi}{\partial u^{2}}>0, \quad \text { and } \quad \frac{\partial^{2} \Phi}{\partial v^{2}}>0 .
$$


Next, we divide (2.8) by (2.7) to obtain a phase space representation of (2.6) and (2.7)

$$
\frac{d u}{d v}=\frac{2(Q-P)}{\partial P / \partial u(u-v)}-\frac{\partial P / \partial v}{\partial P / \partial u} .
$$

Stress-stretch description. We perform one more coordinate change on Eq. (2.10) to recast it in a form which is especially amenable to qualitative analysis. Equation (2.10) is set in the $(u, v)$ plane with $Q$ and $P$ regarded as given functions of the stretches $u$ and $v$. Our final coordinate transform is an interchange of the role of the radial stretch $u$ in favor of the radial stress $P$. This technique is similar to the Von Mises transformation of the two-dimensional compressible boundary layer equations of fluid dynamics (see Von Mises and Friedrichs [7] for details). A new variant of Eq. (2.10), set in the $(P, v)$ plane, is the result.

Before transforming (2.10), we invert Eq. (2.5a) to obtain $u$ in terms of $v$ and $P$. That this is possible is a consequence of (2.9) and the natural growth conditions

$$
P \rightarrow \infty \text { as } u \rightarrow 0, \text { and } P \rightarrow-\infty \text { as } u \rightarrow-\infty \text {. }
$$

The inversion is global in the sense that for every $v>0$ and $P$, there is a negative $u$ given by

$$
u=\hat{u}(P, v) .
$$

With this inversion of $(2.5 \mathrm{a})$ in hand, we proceed to transform (2.10). Multiplying by $\partial P / \partial u$ and rearranging, we have

$$
\frac{\partial P}{\partial u} \frac{d u}{d v}+\frac{\partial P}{d v}=\frac{2(Q-P)}{u-v} .
$$

If we now interpret $u$ in (2.11) via the inversion, the left-hand side is just the total derivative of

$$
P=P(\hat{u}(P, v), v) \equiv \widehat{P}(v)
$$

with respect to $v$. Similarly, we define

$$
Q=Q(\hat{u}(P, v), v) \equiv \widehat{Q}(P, v) .
$$

The transformed equation in the $(P, v)$ plane is

$$
\frac{d P}{d v}=\frac{2(\widehat{Q}-\widehat{P})}{\hat{u}-v}
$$

with the boundary conditions $P=0$ at $r=r_{i}$ and $r=r_{o}$.

In addition to the growth conditions

$$
P \rightarrow \infty \quad \text { as } u \rightarrow 0, \text { and } P \rightarrow-\infty \quad \text { as } u \rightarrow-\infty,
$$

we shall require for our analysis additional growth conditions on $Q$

$$
Q \rightarrow \infty \quad \text { as } v \rightarrow \infty, \text { and } Q \rightarrow-\infty \text { as } v \rightarrow 0,
$$

and we shall require that the discriminant

$$
D \equiv \frac{\partial P}{\partial u} \frac{\partial Q}{\partial v}-\frac{\partial P}{\partial v} \frac{\partial Q}{\partial u}
$$

be positive in $u<0, v>0$. We discuss the latter condition below. 
Qualitative analysis. We now perform the qualitative analysis of Eq. (2.12) that will allow us to state conditions sufficient for existence and uniqueness of solutions. First, we examine the direction of the vector field in the $(P, v)$ plane described by (2.7) and (2.12). We note that the right-hand side of (2.7) is always negative. The right-hand side of the product of (2.7) and (2.12),

$$
\frac{d P}{d v} \frac{d v}{d r}=\frac{2}{r}(\widehat{Q}-\widehat{P})
$$

is either positive or negative, depending on the difference of $Q$ and $P$. In the deformed configuration, the sphere will occupy the thickness between $v=v_{o}$ and $v_{i}$ where $v_{o}$ is the position of the surface $r=r_{o}$ and $v_{i}$ with $r_{i}<r_{o}$ whereas $v_{o}<v_{i}$.

We can prove that the right-hand side of (2.13), and therefore $d P / d r$, has a zero on any $P=$ constant line by appealing to strong ellipticity and the appropriate growth conditions. Computing the derivative of the right-hand side of (2.13) along a line $P=$ constant, we obtain

$$
\begin{aligned}
\frac{\partial}{\partial v}[Q(\hat{u}(P, v), v)-P]_{P=c} & =\frac{\partial Q}{\partial u} \frac{\partial \hat{u}}{\partial v}+\frac{\partial Q}{\partial v} \\
& =\frac{\partial Q}{\partial v}-\frac{\partial Q}{\partial u} \frac{\partial P / \partial v}{\partial P / \partial u}=\frac{D}{\partial P / \partial u} .
\end{aligned}
$$

Strong ellipticity and the growth conditions on $Q$ imply that $Q-P$, a continuous function of $v$, runs through $(-\infty, \infty)$ along the line $P=$ constant between $v=0$ and $v=\infty$. In fact, we can prove that $Q-P$ has a unique zero at some point $v^{*}$ on each half line $v>0, P=$ constant. This follows from the global implicit function theorem, the strong ellipticity property, the growth conditions on $Q$ and $P$, and the requirement $D>0$. Moreover, the fact that $Q$ and $P$ are assumed to be $C^{1}$ implies that $v^{*}(P)$ is a $C^{1}$ function in $v>0$.

In the hyperelastic case, the expression $D>0$ becomes

$$
\frac{\partial^{2} \Phi}{\partial u^{2}} \frac{\partial^{2} \Phi}{\partial v^{2}}-\left[\frac{\partial^{2} \Phi}{\partial u \partial v}\right]^{2}>0 .
$$

Thus $D>0$ corresponds to the positivity of the Hessian of the specialized work function $\Phi(u, v)$. We must carefully distinguish between the positivity of the Hessian of $\Phi$ with respect to the specialized class of deformations presently under consideration, i.e., purely radial eversions, and the positivity of the Hessian of $\sigma(\mathbf{F})$ with respect to all possible three-dimensional deformations. Requirements on the positivity of the Hessian of $\sigma(\mathbf{F})$ lead to several well-known problems (see Marsden and Hughes [4] for a discussion).

It is clear that candidate solution trajectories will begin on the $P$-axis at the point $v=v_{i}$, move on to intersect $v^{*}(P)$ with zero slope, and move back to intersect the $P$-axis at $v=v_{o}$. Thus at the end point $v_{o}, Q<0$ and $P=0$, and at the end point $v_{i}, Q>0$ and $P=0$. Because there are no singular points in the phase plane within $u<0, v>0$, trajectories of (2.7) and (2.13) cannot cross; thus they are ordered by the maximum radial stress achieved through the cross-section, $P_{\max }$, which occurs at some point $\left(P_{\max }, v^{*}\left(P_{\max }\right)\right)$ in the $(P, v)$ plane. The curve $v^{*}(P)$ on which $(Q-P)=0$ is the boundary in the $(P, v)$ plane between the regions where 
$(Q-P)<0$ and $(Q-P)>0$. Examination of Eq. (2.12) shows that through every point on the curve $v^{*}(P)$ with $P<0$, there passes a trajectory which intersects the $v$ axis twice.

We remark that if the condition $D>0$ is violated in $u<0, v>0$, then it is possible that a multiplicity of everted solutions exist for a shell of a given thickness, as we show by the following argument. Consider the case where $D$ is not strictly positive on the $P$-axis in the $(P, v)$ plane. Thus there are, say, $k$ points $\left(0, v_{i}^{*}\right)$ where $Q-P=0$ on the $P$-axis. We assume for simplicity that $D>0$ at these $k$ points in the $(P, v)$ plane. Then by the local implicit function theorem, there exist $k$ curves $v_{i}^{*}(P)$ with $v_{i}^{*}(0)=v_{i}^{*}$. It is possible that for a given shell thickness there is a solution trajectory crossing through $v_{i}^{*}(P)$ (and intersecting the $P$-axis twice) and another crossing through $v_{j}^{*}(P)$ with $i \neq j$. Thus violation of $D>0$ allows for nonuniqueness, i.e., $D>0$ and the conditions on the functions $P$ and $Q$ are sufficient conditions for existence and uniqueness of solutions.

Reference length condition. Finally, we must require that the candidate trajectories intersect the physical boundaries at the appropriate $r_{i}$ and $r_{o}$. This is the reference length condition. Integrating Eq. (2.7), we obtain

$$
\int_{v_{i}}^{v_{o}} \frac{d v}{u-v}=\int_{r_{i}}^{r_{o}} \frac{d r}{r}=-\log \frac{r_{i}}{r_{o}} .
$$

This is the ratio of radii in the reference configuration corresponding to a particular candidate trajectory. To each $r_{i} / r_{o}>0$ there corresponds one and only one trajectory of (2.7) and (2.13) which satisfies the boundary conditions of zero traction on the spherical faces. We prove that the reference length condition provides a 1:1 map between solution trajectories in the first quadrant of the $(P, v)$ plane and ratios of reference length radii $r_{i} / r_{o}$ in $(0,1]$ as follows. To begin, we rewrite $(2.15)$ more explicitly as

$$
g\left(P_{\max }\right) \equiv \log \frac{r_{i}}{r_{o}}=\int_{v_{o}\left(P_{\max }\right)}^{v_{t}\left(P_{\max }\right)} \frac{d v}{\hat{u}\left(\mathscr{P}\left(v ; P_{\max }\right), v\right)-v}
$$

where $\mathscr{P}\left(v ; P_{\max }\right)$ is a particular solution trajectory of $(2.12)$ ordered by its maximum compressive radial stress $P_{\max }$. We note immediately that $g(0)=0$, corresponding to $r_{i} / r_{o}(0)=1$. This means that a shell of vanishing thickness has zero radial stress in the everted state. One can also show that $g \rightarrow-\infty$ as $P_{\max } \rightarrow \infty$, corresponding to $r_{i} / r_{o} \rightarrow 0$, by noting that $\mathscr{P}\left(v, P_{\max }\right) \rightarrow \infty, v_{i} \rightarrow \infty$, and $v_{o} \rightarrow 0$ as $P_{\max } \rightarrow \infty$ because trajectories cannot cross.

Finally, we show $d g / d P_{\max }<0$ for all $P_{\max }>0$ to establish that the map taking $P_{\max }$ to $r_{i} / r_{o}$ is $1: 1$. Differentiating $g$, we have

$$
\frac{d g}{d P_{\max }}=\frac{d v_{i}}{d P_{\max }} \frac{1}{\hat{u}\left(0, v_{i}\right)-v_{i}}-\frac{d v_{o}}{d P_{\max }} \frac{1}{\hat{u}\left(0, v_{o}\right)-v_{o}}-\int_{v_{o}}^{v_{\prime}} \frac{(\partial \hat{u} / \partial P)\left(\partial P / \partial P_{\max }\right)}{(\hat{u}-v)^{2}} d v ;
$$

one observes that each term on the right-hand side is negative.

For reference, we repeat the constitutive restrictions sufficient for existence and uniqueness: the material must be elastic and satisfy the strong ellipticity condition and the growth conditions $Q \rightarrow \infty$ and $v \rightarrow \infty, Q \rightarrow-\infty$ as $v \rightarrow 0, P \rightarrow \infty$ as $u \rightarrow 0$ and $P \rightarrow-\infty$ as $u \rightarrow-\infty$, and have a strictly positive discriminant. 
Example. We give an example of a hyperelastic material which satisfies the hypotheses of Theorem 1, and demonstrate that solutions behave as our qualitative analysis predicts. We choose for the purposes of demonstration a particular case of Ogden material with work function

$$
\sigma(\mathbf{F})=\frac{\alpha}{2}\left(\operatorname{tr} \mathbf{F} \mathbf{F}^{T}-3-2 \log \operatorname{det} \mathbf{F}\right)+\frac{\beta}{2}(\operatorname{det} \mathbf{F}-1)^{2} .
$$

This work function leads to the stresses

$$
\begin{aligned}
& P=\frac{\alpha}{u}\left(u^{2}-1\right)+\beta v^{2}\left(u v^{2}-1\right) \\
& Q=\frac{\alpha}{v}\left(v^{2}-1\right)+\beta u v\left(u v^{2}-1\right)
\end{aligned}
$$

which have the correct growth conditions for the choices $\alpha=1, \beta=1$.

We begin by inverting $(2.17 \mathrm{a})$ to obtain

$$
\hat{u}(P, v)=\frac{\left(P+\beta v^{2}\right)-\sqrt{\left(P+\beta v^{2}\right)^{2}+4 \alpha\left(\alpha+\beta v^{4}\right)}}{2\left(\alpha+\beta v^{4}\right)} .
$$

The quantities appearing in the governing equation (2.12) are known through Eqs. (2.17) and (2.18). Next, we integrate (2.12) numerically, beginning at several sample points on the $P$-axis. The integration is continued until the trajectory again intersects the axis; the result is a particular solution trajectory. Finally, we apply the reference length condition via a numerical integration of (2.15).

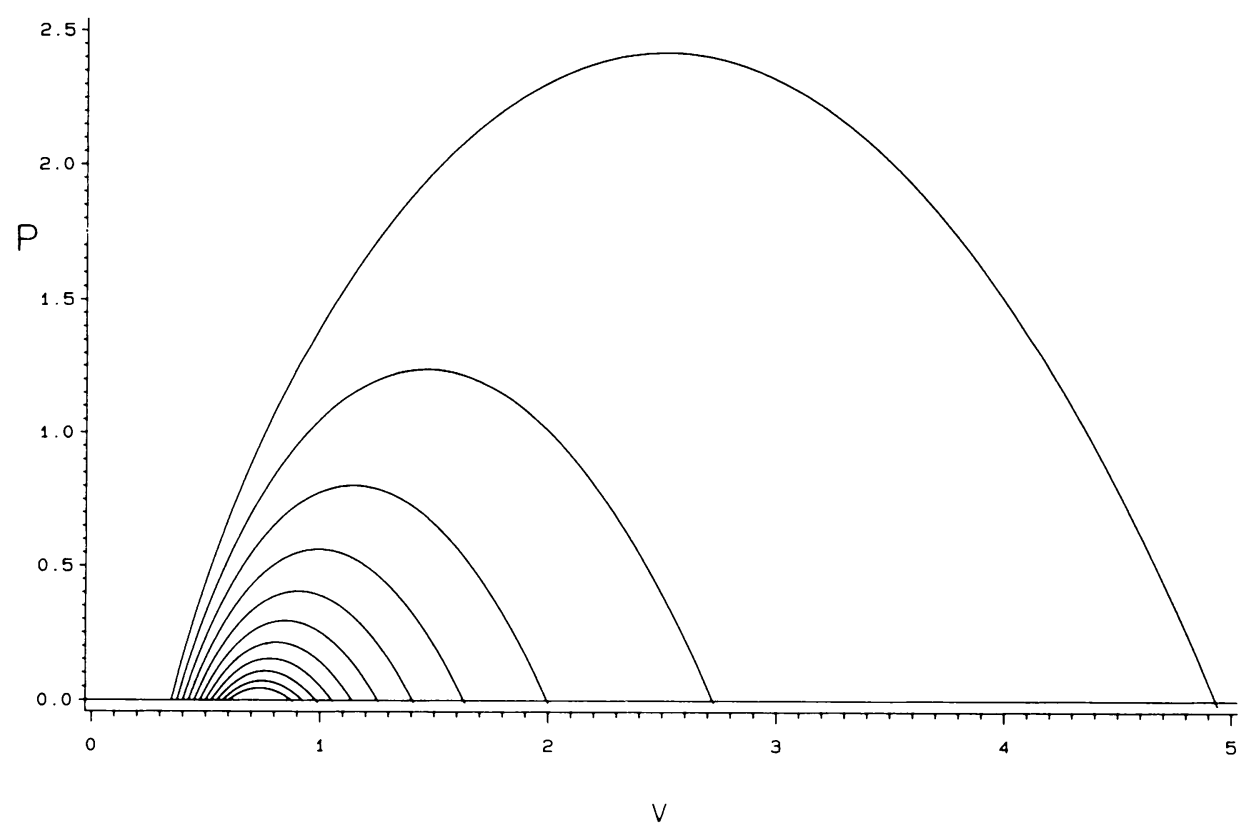

Fig. 1. Each curve represents the radial stress distribution through the thickness of the spherical shell in its everted state. From top to bottom of the figure, the corresponding thicknesses of the shells in the relaxed state are $r_{i} / r_{o}=0.13,0.25,0.35,0.44,0.51,0.58,0.64,0.69$, $0.73,0.78$, and 0.82 . 


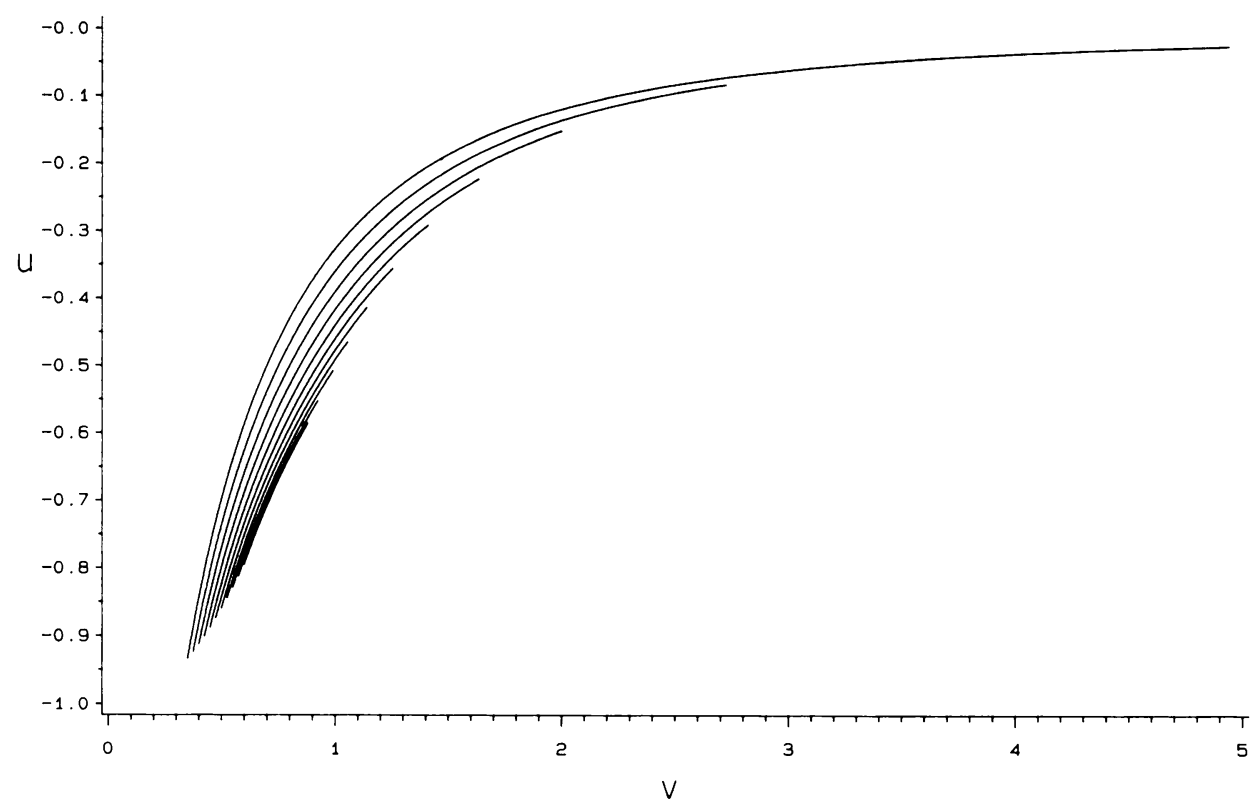

Fig. 2. The trajectories of Fig. 1 recast in phase space. The uppermost trajectory of Fig. 2 corresponds to the uppermost trajectory of Fig. 1, etc.

Figure 1 shows the computed solutions in the stress-stretch plane. Each solution is for a different ratio of radii in the reference configuration, varying from $r_{i} / r_{o}=0.13$ to 0.82 . One observes immediately that larger maximum compressive stresses obtain in thicker spherical shells upon eversion. These same solutions are again depicted in Fig. 2, but in the phase plane. Here the application of the reference length condition is easier to visualize.

3. Eversion of cylindrical shells. The analysis described below is similar to that of Sec. 2. An additional complication arises due to the presence of a third stretch along the axis of the cylinder, but the same ideas apply. The result one can prove is

THEOREM 2. Given a cylindrical shell of an elastic material, there is a unique everted state for the shell provided that the stress functions of the material satisfy the strong ellipticity condition, the appropriate growth conditions, and have a strictly positive discriminant in the radial and azimuthal directions.

Formulation and analysis. We define a cylindrical shell to be

$$
\mathbf{B}=\left\{(r, \theta, z): r_{i} \leq r \leq r_{o}, 0 \leq \theta \leq 2 \pi, z_{1} \leq z \leq z_{2}\right\},
$$

where $(r, \theta, z)$ are cylindrical polar coordinates assigned to positions in Euclidean three-space. In the reference configuration, we have the orthonormal basis vectors

$$
\begin{aligned}
& \mathbf{a}_{1}(\theta)=\mathbf{a}^{1}(\theta)=\cos \theta \mathbf{i}+\sin \theta \mathbf{j} \\
& \mathbf{a}_{2}(\theta)=\mathbf{a}^{2}(\theta)=-\sin \theta \mathbf{i}+\cos \theta \mathbf{j} \\
& \mathbf{a}_{3}=\mathbf{a}^{3}=\mathbf{k} .
\end{aligned}
$$


In the deformed configuration, we have the orthonormal basis vectors

$$
\begin{aligned}
& \mathbf{b}_{1}(\theta)=\mathbf{b}^{1}(\theta)=\cos \theta \mathbf{i}+\sin \theta \mathbf{j} \\
& \mathbf{b}_{2}(\theta)=\mathbf{b}^{2}(\theta)=-\sin \theta \mathbf{i}+\cos \theta \mathbf{j} \\
& \mathbf{b}_{3}=\mathbf{b}^{3}=\mathbf{k} .
\end{aligned}
$$

We consider deformations of the form

$$
\mathbf{p}(r, \theta, z)=f(r) \mathbf{b}_{1}(\theta)+\lambda \mathbf{b}_{3}
$$

where $\lambda$ is a fixed real number. The deformation gradient tensor is

$$
\mathbf{F}=\frac{d f}{d r} \mathbf{b}_{1} \mathbf{a}^{1}+\frac{f}{r} \mathbf{b}_{2} \mathbf{a}^{2}+\lambda \mathbf{b}_{3} \mathbf{a}^{3},
$$

with determinant $f^{\prime}(r) f \lambda / r>0$ for $\mathbf{p}$ to be an orientation preserving map. This implies that $f^{\prime}(r)<0$ and $\lambda<0$ in the case of eversion. In terms of the phase space variables $u \equiv d f / d r$ and $v \equiv f(r) / r$, the radial stress is $P(u, v, \lambda)$ and the azimuthal, or hoop stress is $Q(u, v, \lambda)$.

For equilibrium, we require that the divergence of the stress vanish. This yields the governing equation

$$
\frac{d P}{d r}+\frac{1}{r}(P-Q)=0
$$

which is the analog of (2.6a). The boundary conditions are zero traction on the cylindrical faces, i.e., $P=0$ on $r=r_{i}$ and $r=r_{o}$, and the flat end faces of the cylindrical shell are constrained to lie against lubricated planes perpendicular to the axis of the cylinder. The equation relating $u, v$, and $r$ is again

$$
\frac{d v}{d r}=\frac{u-v}{r} \text {. }
$$

As in Sec. 2, we analyze the governing equations to establish that in each $\lambda=$ constant slice of stress-stretch space the vector field looks like the vector field for the case of the spherical shell. Finally, we assign a fixed negative number to $\lambda$ and compute the reference length via (2.15). As for spherical shells, we prove that the reference length condition is uniquely selective in the sense that it is a $1: 1$ map between the set of trajectories and the ratios $r_{i} / r_{o}$. Different values of $\lambda$ correspond to different tractions and moments that must be applied to the flat end faces of the cylinder to maintain the deformation.

4. Conclusions. We have proved, with the hypothesis of relatively mild constitutive assumptions, that spherical and cylindrical shells of quite general compressible elastic materials have unique everted states. We began the analysis by recasting both problems in stress-stretch coordinates. By this we mean that the roles of the radial component of the first Piola-Kirchhoff stress and the radial stretch are interchanged mathematically. This leaves the governing nonlinear ordinary differential equation in a particularly simple form. Exploitation of the constitutive restrictions and examination of the equation made possible a qualitative understanding of the nature of the vector field described by the equation of equilibrium. Finally, it was observed that there exists a continuum of possible solutions which meet the boundary conditions, 
but that each uniquely corresponds to a particular shell thickness in the reference configuration.

The principal limitation of our analysis is that the solutions are known only qualitatively. For a spherical shell of a given thickness, for example, one must numerically integrate a two-point boundary value problem via a shooting method in order to satisfy the reference length condition.

Acknowledgment. The author gratefully acknowledges the support of the National Science Foundation through the Graduate Fellowship Program and under RCD 8550714. Also helpful were the comments of a referee, and the interest and encouragement of Philip Holmes and Tim Healey.

\section{REFERENCES}

[1] S. S. Antman, The eversion of thick spherical shells, Arch. Rational Mech. Anal. 70, 113-123 (1979)

[2] A. E. Green and J. E. Adkins, Large Elastic Deformations, Clarendon Press, Oxford, 1960

[3] R. Hill, On uniqueness and stability in the theory of finite elastic strain, J. Mech. Phys. Solids 5, 229-241 (1957)

[4] J. E. Marsden and T. J. R. Hughes, Mathematical Foundations of Elasticity, Prentice-Hall, Englewood Cliffs, New Jersey, 1983

[5] J. Sivaloganathan, Uniqueness of regular and singular equilibria for spherically symmetric problems of nonlinear elasticity, Arch. Rational Mech. Anal. 96 (2), 97-136 (1986)

[6] C. Truesdell and W. Noll, The nonlinear field theories of mechanics, Handbuch der Physik, vol. III/3, Springer-Verlag, Berlin, 1965

[7] R. von Mises and K. O. Friedrichs, Fluid Dynamics, Springer-Verlag, New York, 1971 